\title{
Finite Element Analysis of Osteosynthesis Miniplate for the Reconstruction of Parasymphyseal Compound Fracture
}

\author{
Muslim Mahardika", Romario A. Wicaksono", Maria G. Widiastuti", Budi Arifvianto ${ }^{\#}$, and Suyitno \\ ${ }^{*}$ Department of Mechanical and Industrial Engineering, Universitas Gadjah Mada, Yogyakarta, 55281, Indonesia \\ E-mail:muslim_mahardika@ugm.ac.id
}

*Department of Oral Surgery, Dr. Sardjito General Hospital, Yogyakarta, 55281, Indonesia

\begin{abstract}
In the last two decades, the use of osteosynthesis miniplate has been growing to aid the healing process and reconstruction of fractured mandibular bone. In principle, the plate is used to provide stable fixation of the fractured bone tissue during the healing process and reconstruction. Based on earlier studies, it is noted that arrangements and geometry of the osteosynthesis miniplate played a critical role in determining the stability of the fractured mandibular bone, as well as the miniplate. In this research, a simulation with finite element method (FEM) was carried out to investigate the influence of the number of holes in an osteosynthesis miniplate on the stability of fractured mandibular bone and the corresponding miniplate after the implantation. For this purpose, a set of osteosynthesis miniplate with three different configurations was taken for simulation using a three-dimensional (3D) model of mandibular bone generated from the patient through computed tomography (CT). The result of the simulation showed that all the miniplates with three configurations tested were stable enough to prevent movement of fractured mandibular bone. Moreover, fixation with a pair of miniplates having four screw holes demonstrated the desired result; as indicated by the lowest value of displacement, pressure on the bone surface and pressure on the miniplate.
\end{abstract}

Keywords - miniplate; FEA; parasymphyseal compound fracture

\section{INTRODUCTION}

Technology for treating bone fracture has been growing continuously since more than 5000 years ago [1]. At the ancient time, natural ingredients such as oil and honey were used to treat patients with several cases of fractures. Later, the Greek physician, Hippocrates, introduced horizontal wiring technique to realign the fractured bone with cerate to keep the fractured bone remained at its position. This technology had been used for the last five decades when the internal fixation-based miniplate was first introduced. Since then, techniques for treating fractures are further developed to reduce the healing time and to improve the outcomes of surgery.

Up till now, fracture of a mandibular bone is one of the major concerns in oral surgery as well as in their relevant research [2]. Complexities of mandibular bone geometry led to the difficulties in generating the model of this bone for a numerical simulation study. In the last two decades, the emergence of imaging technology, such as computed tomography (CT) or magnetic resonance imaging (MRI), had led to significant advancements in medical technique development [3].

This technology has been highly developed and reliable for various needs in the medical application. Imaging data allows the surgeon to develop the treatment procedure in even more complicated conditions [4]. To date, medical imaging data is most commonly used in branches of surgery involving the musculoskeletal system, such as oral and maxillofacial surgery, traumatology, and orthopedic surgery. The CT data can then be used for the fabrication of drill guides, saw guides, and patient-specific implants [5]. The CT image also represents an advance with regard to the various stages of reconstructive surgery planning, but also in the production of prototype surgical instruments, Maxillofacial prostheses, and surgical guides [6].

In this case, data of particular bone from a patient retrieved from CT or MRI scanning are used in the simulation with finite element method (FEM) [7], [8]. With FEM, the effect of mechanical loading on the complex mechanical behavior of mandibular bone can, for instance, be simulated and predicted [9], [10].

In an attempt to design a miniplate for osteosynthesis with optimum performance, FEM could be used to deal with the challenge in overcoming the complexity of mandibular bone geometry. Basically, in their application, an osteosynthesis miniplates should be able to provide stable fixation for the fractured mandibular bone, so that an excessive motion of fractured bone that may cause delayed healing process could 
be avoided [11]. A proper fixation should be able to keep the fracture gap below its critical value. Nowadays, clinicians established a maximum acceptable fracture gap of $1 \mathrm{~mm}$ before reconstruction to allow bone recovery. A larger fracture gap will lead to secondary bone healing and the formation of a fibrocartilaginous intermediary bone callus [12]. With a proper fixation, the formation of callus bone could be minimized [13]. The presence of external callus indicated an improper healing process and could lead to a slower the formation of new bone at the fracture site.

Basically, mechanical interlocking between fragments or surface of fractured bones contributed to stabilizing the fixation. However, mandibular loading which can lead led to mechanical interlocking of the surface of fractured bone could hardly be predicted. Therefore, an osteosynthesis miniplate is needed to stabilize the fixation and achieve primary bone healing of the fractured bone [14]. Moreover, to improve the healing process, a two-miniplate configuration is required [15], [16].

In this research, a simulation with finite element method (FEM) was carried out to investigate the influence of the number of holes in an osteosynthesis miniplate on the stability of fractured mandibular bone and the corresponding miniplate after the implantation. In addition, as the miniplate may behave differently under tension and compression loads, the number of screws used for the implantation of this plate should be determined appropriately [17].

\section{MATERIALS AND METHODS}

In this research, a set of osteosynthesis miniplates with three different screw configurations were used in the fixation of fractured mandibular bone. All these configurations were simulated by using finite element method (FEM) to evaluate (1) stability of fixation and (2) pressure distribution on the miniplate. The three configurations, namely MF-42, MF-44, and MF-24, could be distinguished from the number of screws used to implant the miniplate and the arrangements of the miniplate on the fractured mandibular bone. In MF-42 configuration, a miniplate with four screws were applied at the top location in the mandible, while a miniplate with two screws was implanted below the 4-screws miniplate. In MF44 configuration, both the top and bottom miniplates were designed with four screws. Finally, in MF-24 configuration, a miniplate with two screws was implanted at the top location in the mandible, while the one with four screws was placed below the 2-screws miniplate. These three configurations applied to minimize the fracture gap on parasymphyseal side of a 3-dimensional (3-D) mandibular bone model, based on Champy's line [18].

\section{A. Model Generation}

In this research, a 3-D mandibular model was generated from CT scan images of a patient with a mandibular bone fracture. The image of the mandible was separated as a single mask through extraction of ROI (region of interest). 3-D stereolithography (.stl) file format model was used with a triangular mesh that was generated based on the previously created masks for surface representation in a solid mandible. Once the CT-scan image was converted into a .stl file, the 3$\mathrm{D}$ mandible could be reconstructed into a single unit of surface. Afterwards, fracture of mandibular bone was modeled by using Autodesk Meshmixer 3.2 (Autodesk, USA), by separating the model into two bone pieces with fracture line. Smoothing and re-meshing were also applied to produce high-quality images of triangular meshes.

Fixation of fractured mandibular bone with osteosynthesis miniplates was modeled by using Inventor Professional 2017 and Fusion 360 software (Autodesk, USA). At this step, the miniplate was placed at the surface of a 3-D model of the mandible by using Inventor Professional 2017. Screws were also inserted into the bone model and miniplate; making two fragments to be fixed together during the FEM simulation.

\section{B. FEM Modelling}

In this research, the simulation was carried out by using a FEM software, i.e., Abaqus (V6.11, Dassault Systems, Cedex, France), to create tetrahedron meshes in the model. The mesh of the mandible and miniplate were modeled with triangular mesh; consisting of 61,387 elements for the mandible model, 621 elements for the miniplate model having two screws, 1,675 elements for the miniplate with four screws.

\section{Material Properties and Model}

Despite non-homogeneous, the density of mandibular bone in this research is considered of constant with values ranging from 1.85 to $2 \mathrm{~g} \mathrm{~cm}-3$ [14]. For the mandibular bone, an elastic modulus of $22.8 \mathrm{GPa}$ and Poisson's ratio of 0.18 was used in the computation of this research [19]. The osteosynthesis miniplate was made from pure titanium with an elastic modulus of $102.1 \mathrm{GPa}$, and the Poisson ratio is 0.8 [20], while the fixation screws were made from Ti-6Al-4V alloy with an elastic modulus of $112 \mathrm{GPa}$ and the Poisson ratio of 0.37 [20].

TABLE I

Bite Force on PAtients (N) [16].

\begin{tabular}{|c|ccc|ccc|ccc|}
\hline & \multicolumn{3}{|c|}{ Male } & \multicolumn{3}{c|}{ Female } & \multicolumn{3}{c|}{ Mean } \\
\hline Preoperative & 293.2 & \pm & 162.0 & 208.5 & \pm & 98.8 & 242.4 & \pm & 131.6 \\
\hline 2 weeks & 60.8 & \pm & 41.5 & 69.6 & \pm & 29.4 & 66.5 & \pm & 33.4 \\
\hline 4 weeks & 127.5 & \pm & 55.4 & 129.7 & \pm & 53.4 & 128.8 & \pm & 53.0 \\
\hline 8 weeks & 184.7 & \pm & 89.0 & 199.1 & \pm & 88.5 & 193.7 & \pm & 87.0 \\
\hline 3 months & 245.9 & \pm & 97.9 & 251.1 & \pm & 107.8 & 249.1 & \pm & 101.8 \\
\hline 6 months & 351.0 & \pm & 256.9 & 276.7 & \pm & 135.1 & 301.5 & \pm & 101.5 \\
\hline Normal & 721.0 & \pm & 505.5 & 530.7 & \pm & 204.6 & 625.9 & \pm & 387.9 \\
\hline
\end{tabular}

Note: Data are mean \pm SD 


\section{Loading and Constrain}

As studied by Harada et al. [21], the bite force decreased dramatically due to the fracture of the mandibular bone, such as shown in Table 1. In their study, Harada et al. [21] examined both bite force and occlusal contact area by using a pressure-sensitive sheet in patients at 2 weeks until 6 months post-surgery. The lowest bite force of the patients appeared in 2 weeks after surgery, i.e., about $66.5 \mathrm{~N}$ and this value increased till $301.5 \mathrm{~N}$ after 6 months. In this research, the bite force used in the simulation was considered constant and selected from the study of Harada et al. [16] at 2 weeks' period after surgery, where the stability of the fractured bone depends entirely on the miniplate used in the fixation.

During the simulation, the surface of the fractured bone model was free from movement and did not contact one another. Also, interfaces of plate-to-bone, plate-to-screw, and screw-to-bone were designed not to separate one another to prevent relative motions. Finally, Von Mises stresses and mandibular displacement during occlusal loading were calculated as the outcome of the simulation. The configuration of the mandibular bone and osteosynthesis miniplates is modeled such as in Fig. 1.

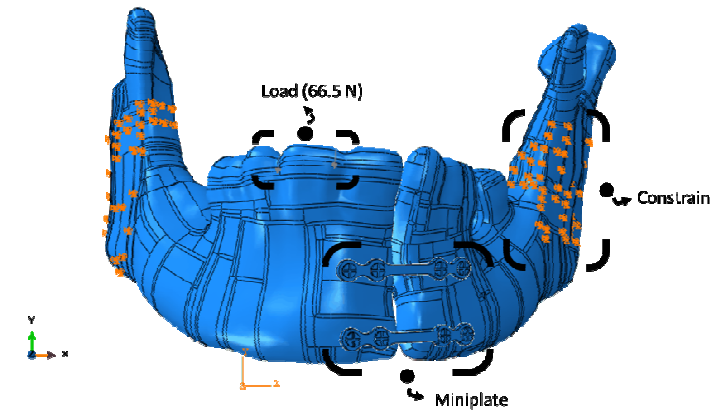

Fig. 1 Loading and boundary condition setup in simulation

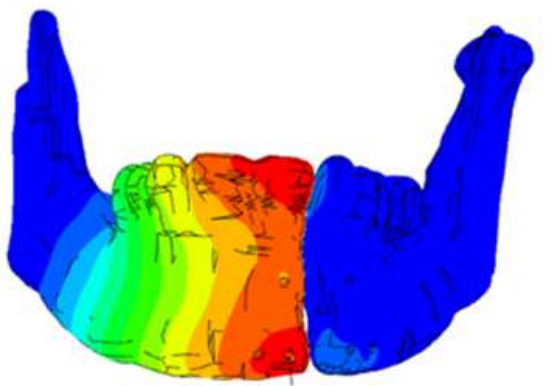

Max: $2.932 \mathrm{e}-02 \mathrm{~mm}$

(a)

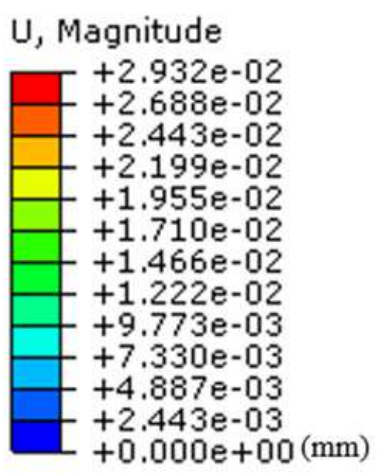

\section{RESULTS AND DISCUSSION}

In this study, two main parameters were studied to determine the performance of osteosynthesis miniplate used in the simulation: (1) fracture gap and displacement of the fractured bone as the application of miniplate, and (2) Von Misses stresses which indicated traction force on the mandible surface. The distance of the fracture gap will determine healing process on the fractured bone, in which the smallest gap between the bone fragments will lead to the highest stability of the fractured bone during the fixation [14]. Moreover, stable fixation is required to facilitate osteogenesis and bone healing. Von Misses stresses were determined to indicate pressure distribution over the bone surface; which in the end could also be used to evaluate how far the fixation system would damage the bone. Based on a previous histological examination, it is confirmed that too high pressure on the mandibular bone surface would damage osteoblast and lead to the formation of callus [13], which in the end retarding the healing process and thus prolong the recovery time of the fractured mandibular bone.

\section{A. Stability of Fixation}

Fig. 2 shows the result of simulation to investigate displacement of fractured mandibular bone that occurred as a result of biting at 2 weeks after surgery. In this simulation, fixation with three different configurations was examined. The healing time of wound due to bone fracture bone is strongly dependent on the stability of the fractured bone. Therefore, a configuration of miniplate that could give the most stable fixation of the fractured mandibular bone should be determined.

As demonstrated in Fig. 2, all configurations of fixation resulted in the displacement of fractured mandibular bone with a length of less than $30 \mu \mathrm{m}$. In previous work, it is revealed that displacement of fractured bone that resulted in a gap $150 \mu \mathrm{m}$ was considered as critical; a gap of larger than

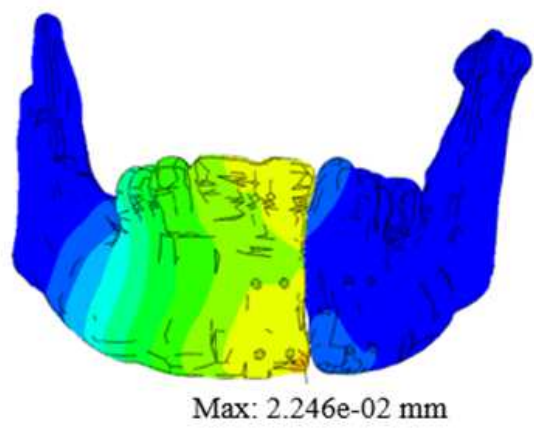

(b)

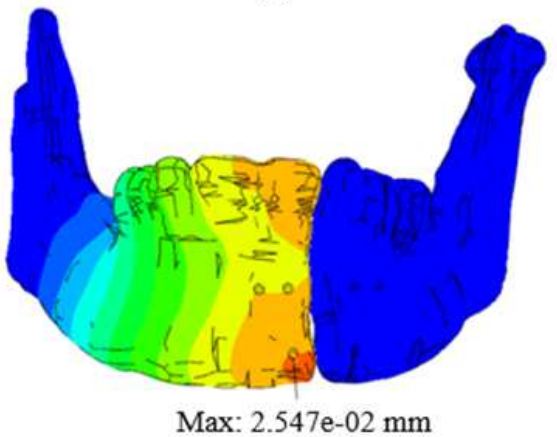

(c)

Fig. 2 Displacement of along the system from (a) MF-24, (b) MF-44, and (c) MF-42 
$150 \mu \mathrm{m}$ might disturb the healing and reconstruction process of the fractured bone [22]. Fixation with MF-24 configuration was found to be the most unstable fixation compared with those with other configurations. Despite the presence of fracture gap, fixation with MF-44 configuration demonstrated the most stable system compared to the other configurations. Nevertheless, on the basis of this simulation, all the configurations of fixation could provide good stability of the fractured mandibular bone during its healing and reconstruction process.

\section{B. Bone Damage}

After surgery, the mandibular bone actually suffered further damage as a result of the implantation of osteosynthesis miniplate. Fixation of the fractured bone by the miniplate generated pressure on bone fracture surface [13] and the area surrounding the hole prepared for the fixation screw. Too high pressure would damage the bone cells and retard the healing process. Furthermore, the bone damage would, in the end, cause the formation of callus. Therefore, bone damage at the area surrounding the holes for fixation screws as a result of overpressure during the fixation should be avoided.

Fig. 3 shows the pressure in the holes of screws that were used to keep the fractured bone together during the healing and reconstruction process. A bite force of $66.5 \mathrm{~N}$ was applied in the simulation, and the pressure distribution in the area surrounding the screw hole of the miniplate as the reaction of this load was determined. It is shown in Fig. 3 that the inferior minipate system resulted in higher pressure around the screw hole, as compared to the superior miniplate. The highest pressure occurred in the screw holes with MF24 configuration. Meanwhile, the MF-44 configuration the lowest pressure around the screw hole.

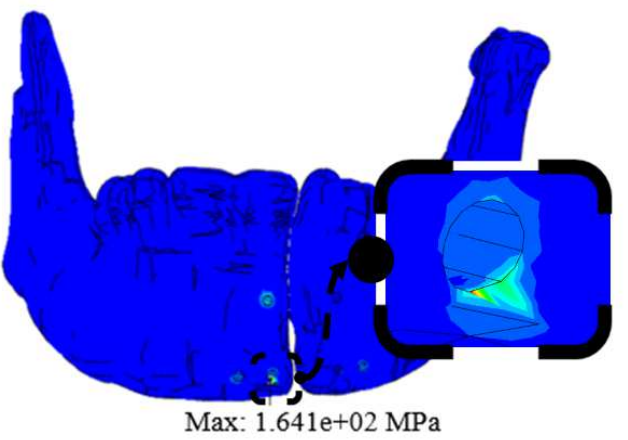

(a)

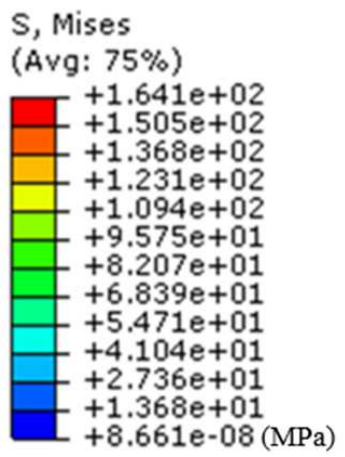

\section{Pressure on Miniplate}

To provide external support for fixation of the fractured mandibular bone, the osteosynthesis miniplate should be able to maintain the fixation of the fractured bone with sufficient strength. Once implanted to the mandibular bone, the miniplate received some portions of forces from the mandible. Fig. 4 shows pressure distribution on the miniplate, as a result of forces transmitted from the fractured mandibular bone.

As shown in Fig. 4, the highest pressure could be observed in regions surrounding the holes in the miniplate. A higher pressure could be seen in a miniplate with a fewer number of holes, such as shown in MF-24 and MF-42 configurations, where a maximum pressure of $3 \mathrm{GPa}$ could be recognized in figures $4 \mathrm{a}$ and $4 \mathrm{c}$. Meanwhile, the pressure on miniplates with MF-44 configuration was three times lower than those in MF-24 and MF-42 configurations. In the plate with MF-44 configuration, pressures were well distributed in all the holes for fixation screws. An osteosynthesis miniplate with 4 screws demonstrated a higher pressure at screw holes located in the middle of the miniplate.

\section{CONCLUSIONS}

In this research, a set of miniplates implanted with three different screw configurations was studied numerically by using finite element method (FEM). The result of the simulation shows that all the miniplates with three configurations tested were stable enough to prevent movement of fractured mandibular bone for more than 150 $\mu \mathrm{m}$ in the distance. Fixation with MF-24 configuration was found to be the most unstable fixation compared with those with other configurations. Moreover, the fixation with MF24 configuration also resulted in the highest pressure on the

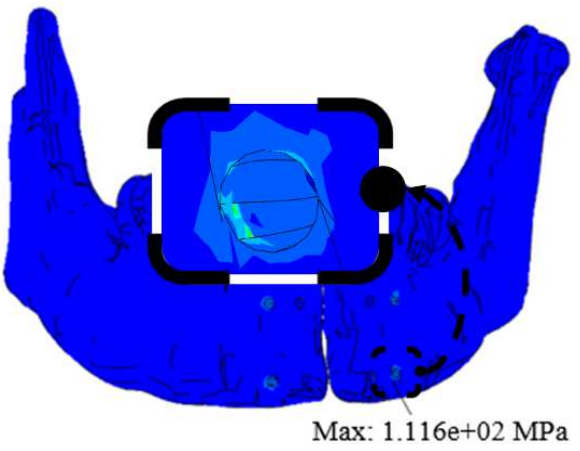

(b)

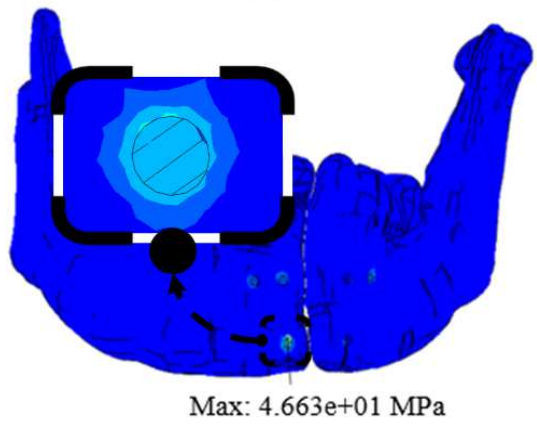

(c)

Fig. 3 Pressure occur in screw hole on bone surface by (a) MF-42, (b) MF-44, and (c) MF-24 configuration 

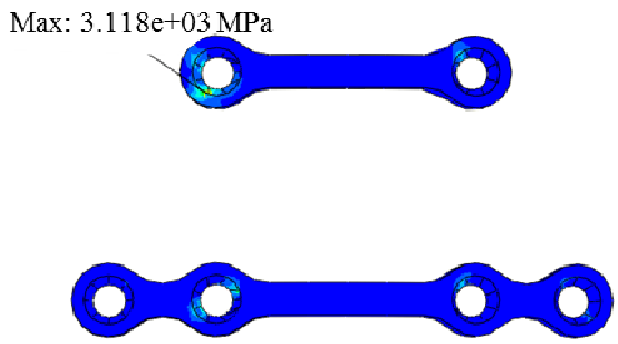

(a)

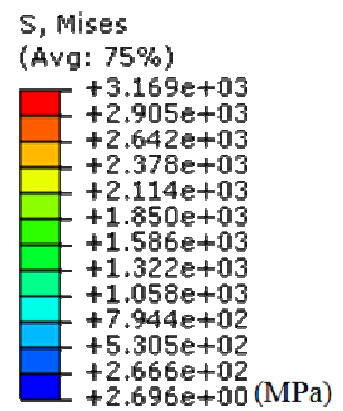

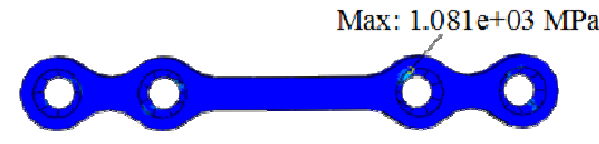

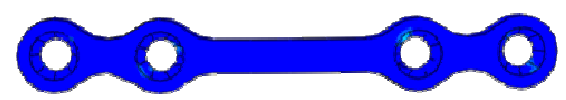

(b)
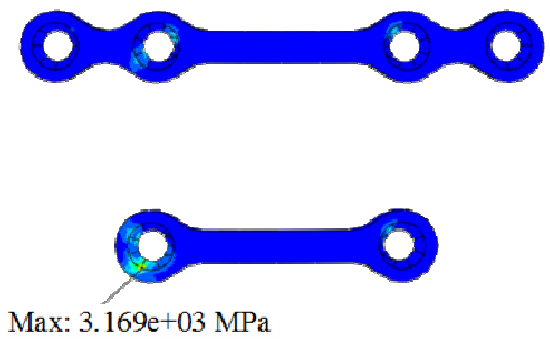

(c)

Fig. 4 Pressure on miniplate as the result of loading by (a) MF-42, (b) MF-44, and (c) MF-24 configuration

bone surface, which subsequently could lead to damage to the bone. Fixation with MF-44 configuration demonstrated the desired result; as indicated by the lowest value of displacement, pressure on the bone surface and pressure on the miniplate.

\section{REFERENCES}

[1] Thoma, K. H., "A historical review of methods advocated for the treatment of jaw fractures, with ten commandments for modern fracture treatment.” Am. J. Orthod. Oral Surg., vol. 30(8), pp. C399C504, 1944.

[2] N.H. Al-Tairi, M.M. Shoushan, M.M. Saad Khedr, S.E. Abd-alal, "Comparison of three-dimensional plate versus double miniplate osteosynthesis for treatment of unfavorable mandibular angle fractures." Tanta Dental Journal, vol. 12, 89-98, 2015.

[3] Y. Arai, E. Tammisalo, K. Iwai, K. Hashimoto, and K. Shinoda, "Development of a compact computed tomographic apparatus for dental use," vol. 240, no. April, pp. 245-248, 1999.

[4] L. Ganry, B. Hersant, J. Quilichini, P. Leyder, J.P. Meningaud, "Use of the 3D surgical modelling technique with open-source software for mandibular fibula free flap reconstruction and its surgical guides," J Stomatol Oral Maxillofac Surg., vol. 118, pp. 197-202, 2017.

[5] M. van Eijnatten, R. van Dijk, J. Dobbe, G. Streekstra, J. Koivisto, J. Wolff, "CT image segmentation methods for bone used in medical additive manufacturing," Medical Engineering and Physics, vol. 51, pp. 6-16, 2018.

[6] L. Ganry, J. Quilichini, C. M. Bandini, P. Leyder, B. Hersant, J. P. Meningaud, "Three-dimensional surgical modelling with an opensource software protocol: study of precision and reproducibility in mandibular reconstruction with the fibula free flap," Int. J. Oral Maxillofac. Surg., vol. 46, pp. 946-957, 2017.

[7] R. T. Hart, V. V Hennebel, and N. Thongpreda, "Modeling the biomechanics of the mandible: a three-dimensional finite element study *,"J. Biomech., vol. 25, no. 3, pp. 261-286, 1992.

[8] T. Silagieva, A. Bucur, C. Gudas, S. Pituru, and O. Marius, "New miniplate for osteosynthesis of mandibular angle fractures designed to improve formation of new bone," J. Cranio-Maxillofacial Surg., vol. 44, no. 4, pp. 500-505, 2016.

[9] N. Narra et al., "Finite element analysis of customized reconstruction plates for mandibular continuity defect therapy," J. Biomech., vol. 47, no. 1, pp. 264-268, 2014.

[10] S. Albougha, K. Darwich, M. A. Darwich, M. H. Albogha, "Assessment of sagittal split ramus osteotomy rigid internal fixation techniques using a finite element method." Int. J. Oral Maxillofac. Surg, vol. 44, pp. 823-829, 2015.

[11] B. Spiessl, "Principles of rigid internal fixation in fractures of the lower jaw," in new concepts in maxillofacial bone surgery, Springer, 1976, pp. 21-34.

[12] R. Wang, Y. Liu, J. H. Wang, and D. A. Baur, "Effect of interfragmentary gap on the mechanical behavior of mandibular angle fracture with three fixation designs: A finite element analysis," J. Plast. Reconstr. Aesthetic Surg., vol. 70, no. 3, pp. 360-369, 2016.

[13] R. Gutwald, R. Scho, M. Metzger, K. Kreutzer, B. Rahn, and R. Schmelzeisen, "Miniplate osteosynthesis with four different systems in sheep," pp. 94-102, 2011.

[14] H. H. Korkmaz, "Evaluation of different miniplates in fixation of fractured human mandible with the finite element method," Oral Surgery, Oral Med. Oral Pathol. Oral Radiol. Endodontology, vol. 103 , no. 6, pp. 20-22, 2007.

[15] R. Wang, Y. Liu, J. H. Wang, and D. A. Baur, "Effect of interfragmentary gap on the mechanical behavior of mandibular angle fracture with three fixation designs: A finite element analysis," J. Plast. Reconstr. Aesthetic Surg., vol. 70, no. 3, pp. 360-369, 2017.

[16] S. George, M. Wolf, R. Wendlandt, H. Kimmerle, P. Sieg, and H. Jacobsen, "Comparative biomechanical study on three miniplates osteosynthesis systems for stabilisation of low condylar fractures of the mandible," Br. J. Oral Maxillofac. Surg., vol. 52, no. 4, pp. 317322, 2014.

[17] B. Ji, C. Wang, L. Liu, J. Long, and W. Tian, "A biomechanical analysis of titanium miniplates used for treatment of mandibular symphyseal fractures with the," YMOE, vol. 109, no. 3, pp. e21-e27, 2010.

[18] M. Champy, J. P. Loddi, R. Schmitt, J. H. Jaeger, and D. Muster, "Mandibular Osteosynthesis by Miniature Screwed Plates via a Buccal Approach *," vol. 17, no. September 1976, pp. 14-21, 1978.

[19] C. L. Schwartz-Dabney and P. C. Dechow, "Variations in cortical material properties throughout the human dentate mandible," Am. J. Phys. Anthropol., vol. 120, no. 3, pp. 252-277, 2003.

[20] M. Ninomi, "Mechanical properties of biomedical titanium alloys," Mater. Sci. Eng. A, vol. 243, no. 1-2, pp. 231-236, 1998.

[21] K. Harada, M. Watanabe, K. Ohkura, and S. Enomoto, "Measure of bite force and occlusal contact area before and after bilateral sagittal split ramus osteotomy of the mandible using a new pressure-sensitive device: a preliminary report," J. oral Maxillofac. Surg., vol. 58, no. 4, pp. 370-373, 2000.

[22] J. Tams, J.-P. Van Loon, B. Otten, and R. R. M. Bos, "A Computer Study of Biodegradable Plates for Internal Fixation of Mandibular," J. Oral Maxillofac. Surg., vol. 59, pp. 404-407, 2001. 